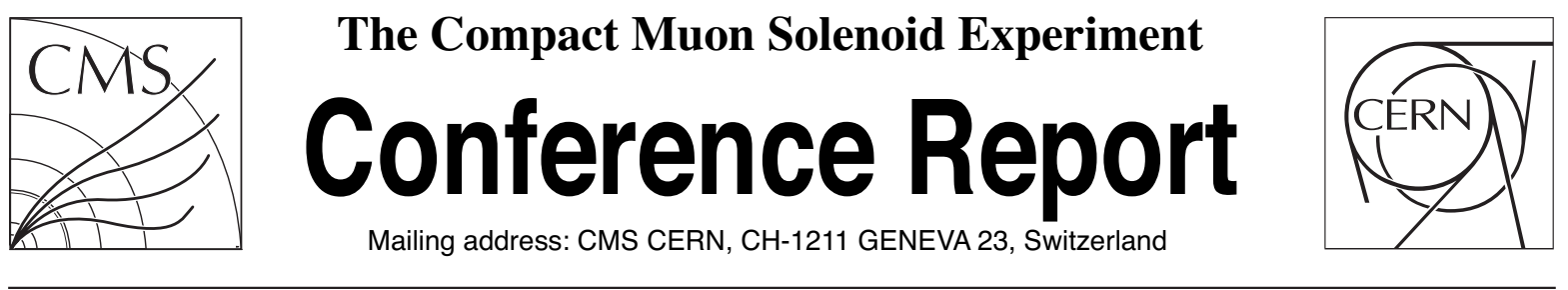

12 November 2009 (v2, 15 November 2009)

\title{
The CMS all silicon Tracker simulation
}

\author{
Maurizio Biasini, Vesna Cuplov, for the CMS Collaboration
}

\begin{abstract}
The Compact Muon Solenoid (CMS) tracker detector is the world's largest silicon detector with about $201 \mathrm{~m}^{2}$ of silicon strips detectors and $1 \mathrm{~m}^{2}$ of silicon pixel detectors. It contains 66 millions pixels and 10 million individual sensing strips. The quality of the physics analysis is highly correlated with the precision of the Tracker detector simulation which is written on top of the GEANT4 and the CMS object-oriented framework. The hit position resolution in the Tracker detector depends on the ability to correctly model the CMS tracker geometry, the signal digitization and Lorentz drift, the calibration and inefficiency. In order to ensure high performance in track and vertex reconstruction, an accurate knowledge of the material budget is therefore necessary since the passive materials, involved in the readout, cooling or power systems, will create unwanted effects during the particle detection, such as multiple scattering, electron bremsstrahlung and photon conversion. In this paper, we present the CMS Tracker simulation description. The simulation was validated comparing its results with CMS cosmic data taken in fall 2008.
\end{abstract}

Presented at IEEE2009: 2009 IEEE Nuclear Science Symposium and Medical Imaging Conference 


\title{
The CMS All Silicon Tracker Simulation
}

\author{
M. Biasini, University and INFN Perugia, Italy and V. Cuplov, Rice University, USA \\ on behalf of CMS collaboration
}

\begin{abstract}
The Compact Muon Solenoid (CMS) tracker detector is the world's largest silicon detector with about $201 \mathbf{~ m}^{2}$ of silicon strips detectors and $1 \mathrm{~m}^{2}$ of silicon pixel detectors. It contains 66 millions pixels and 10 million individual sensing strips. The quality of the physics analysis is highly correlated with the precision of the tracker detector simulation which is written on top of the GEANT4 and the CMS object-oriented framework. The hit position resolution in the tracker detector depends on the ability to correctly model the CMS tracker geometry, the signal digitization, calibration and inefficiency. In order to ensure high performance in track and vertex reconstruction, an accurate knowledge of the material budget is therefore necessary since the passive materials, involved in the readout, cooling or power systems, will create unwanted effects during the particle detection, such as multiple scattering, electron bremsstrahlung and photon conversion. In this paper, we present the CMS tracker simulation description. The simulation was validated by comparing its results with CMS cosmic data taken in fall 2008.
\end{abstract}

CERN, CMS, Tracker, Simulation, Cosmics.

\section{INTRODUCTION}

$\mathbf{T}$ HE CMS tracker[1] is entirely based on silicon detectors. It consists of about $201 \mathrm{~m}^{2}$ of silicon strip detectors and $1 \mathrm{~m}^{2}$ of silicon pixel detectors. Fig. 1 shows the CMS detector and its global coordinate system.

The pixel detector is part of the tracking system, which is closest to the interaction point. It covers a pseudo-rapidity range of $|\eta|<2.4$, which is the geometric acceptance of the central tracker. It is organized into three $53 \mathrm{~cm}$ long cylindrical barrel layers (Pixel Barrel=PXB), positioned at radii of 4.4, 7.3 and $10.2 \mathrm{~cm}$, and two disks per each side (Pixel Forward=PXF), placed in $\mathrm{z}$ at $\pm 35.5 \mathrm{~cm}$ and $\pm 48.5 \mathrm{~cm}$ from the nominal interaction point, covering radii between 6 and $15 \mathrm{~cm}$ to guarantee at least two crossed layers per track. The CMS tracker silicon microstrip sub-detector covers a tracking volume up to a radius of $1.2 \mathrm{~m}$ with a length of $5.6 \mathrm{~m}$ and is organized in three parts: the inner tracker with four barrel layers (Tracker Inner Barrel=TIB) and three disks per endcap (Tracker Inner Disks=TID), six outer barrel layers (Tracker Outer Barrel=TOB) closed by nine wheels on both sides (Tracker End-Cap=TEC). The rz view of the tracker is shown in Fig. 2. The total number of active and passive volumes is summarized in Table I. The CMS tracker offers a very high precision measurement points which is required for charged track pattern recognition together with the CMS 4T magnetic field. The simulation of the CMS tracker is done using GEANT4[2] and CMS software framework[3]. It requires a detailed description of the detector components and materials, a realistic description of the charge deposit and the correct modeling of the detector electronics response (digitization).

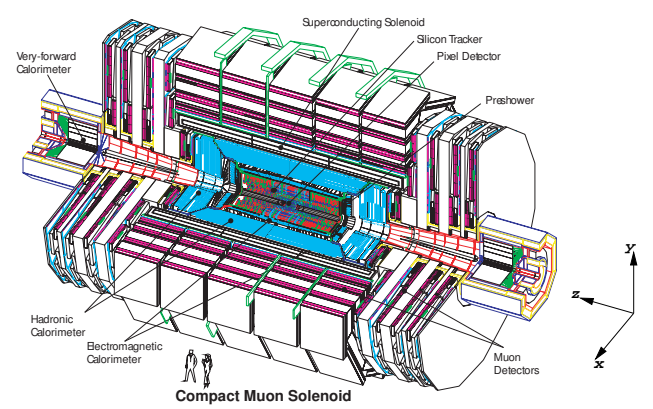

Fig. 1. Layout of the CMS detector including the pixel and strip detectors: Barrel pixel (BPIX) and endcap pixel disks (FPIX), inner barrel (TIB), inner disks (TID), outer barrel (TOB) and endcap (TEC). The CMS global coordinate system is shown on the right.

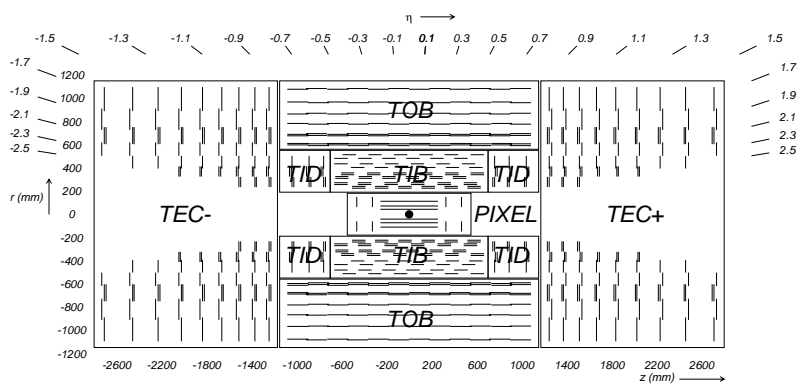

Fig. 2. View of the CMS silicon tracker in the $(r, z)$ plane.

Fig. 3 is a photograph of the interior of one half of the tracker inner barrel (TIB) showing many of the silicon sensors. Fig. 4 is a photograph of one of the eight pixel half disk fully populated with modules and detail of the barrel mechanics partially equipped with modules and connected to the supply tube electronics via flat cables.

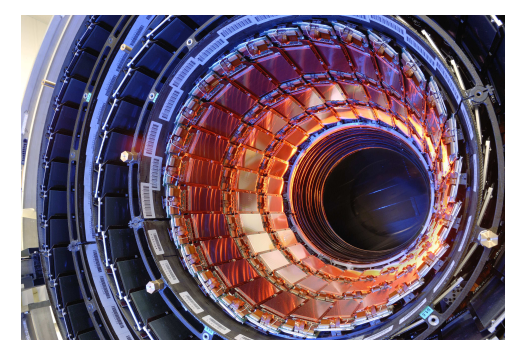

Fig. 3. Photograph of the interior of one half of the tracker inner barrel (TIB) showing many of the silicon sensors. 
TABLE I

NUMBER OF GEANT4 VOLUMES NEEDED TO CONSTRUCT THE TRACKER GEOMETRY. NUMBERS ARE REPORTED PER SUBDETECTORS AND THE TOTAL IS GIVEN FOR THE PIXEL AND MISCROSTRIP DETECTORS AND THE FULL CMS TRACKER.

\begin{tabular}{|c||c||c|}
\hline Subdetector & active volumes & passive volume \\
\hline Pixel Barrel (PXB) & 768 & 10201 \\
Pixel Forward (PXF) & 672 & 23670 \\
\hline PIXEL & 1440 & 33871 \\
\hline \hline Inner Tracker (TIB and TID) & 3540 & 56488 \\
Outer Barrel (TOB) & 5208 & 145419 \\
Outer End-caps (TEC) & 6400 & 113158 \\
Outer structures & 0 & 346 \\
\hline \hline MICROSTRIPS & 15148 & 315411 \\
\hline TRACKER & 16588 & 349283 \\
\hline
\end{tabular}
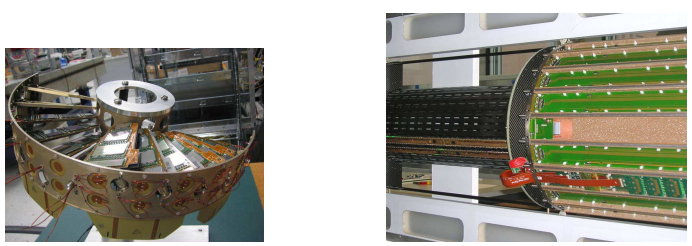

Fig. 4. Photograph of one of the eight pixel half disk (left) fully populated with modules and detail of the barrel mechanics (right) partially equipped with modules and connected to the supply tube electronics via flat cables.

\section{DETECTOR GEOMETRY}

Simulation and Reconstruction have different geometry requirements in CMS. The simulation geometry requires all parts (sensitive and passive) to be described in detail whereas the reconstruction geometry requires only a detailed description of those active parts of the detector that are needed to define hits and tracks and an averaged description of the passive components.

The previous version of the tracker geometry simulation was written using the GEANT3 simulation toolkit [4]. It was not a flexible description and knowing that some components of the detector were not yet designed, the CMS collaboration decided to re-write the geometry in $\mathrm{C}++$ /GEANT4 using the Detector Description Language (DDL) [5] based on eXtensive Markup Language (XML) schema. This description is easily extendable. Fig. 5 shows an IGUANA [6][7] visualization of one layer of the tracker outer barrel with all modules. Fig. 6 and 7 show an IGUANA visualization of one of the forward pixel disks and the pixel barrel layers. The CMS detector is described using volumes which assign a material to a shape. The CMS detector description uses a geometrical hierarchy defined by volumes and placements, which associates two volumes in a parent-child relationship with a relative geometrical transformation between their local reference frames. Through the hierarchy of volumes and materials the generated particles are traced, and the physics processes that accompany particle passage through matter are modeled. Results of each particle's interactions with matter are recorded in the form of simulated hits.

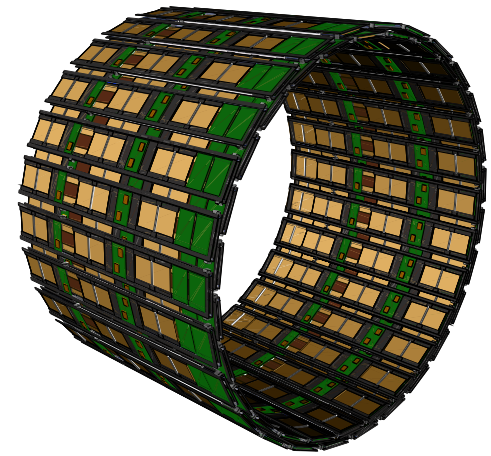

Fig. 5. IGUANA visualization of one layer of the tracker outer barrel.

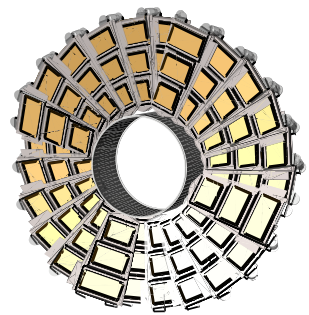

Fig. 6. IGUANA visualization of one of the forward pixel disks. The outer support ring is not shown on the picture.

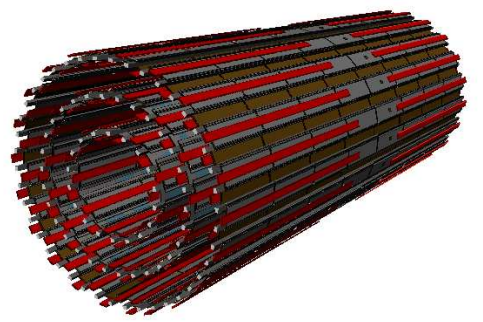

Fig. 7. IGUANA visualization of the pixel barrel. Supply tubes are not shown on the picture.

\section{CHARGE DEPOSIT AND DETECTOR RESPONSE}

In the CMS software framework, the passage of particles through matter is simulated by the GEANT4 toolkit. The amount of energy deposited in each tracker sensitive volume is estimated by subdividing each track segment into small (compared to the sensor thickness) sub-segments of equal size. For each sub-segment the fraction of the deposited energy is fluctuated by the GEANT4 routine G4UniversalFluctuation. This is followed by a digitization step in which the charge from each track sub-segment is drifted to the detector surface and simultaneously diffused in the perpendicular plane. The length of the Lorentz drift in a $3.8 \mathrm{~T}$ magnetic field is calculated by 
the drift length and the Lorentz angle. The charge distribution is then mapped to the tracker geometry and a list of hit channels for all contributing tracks is determined. To simulate the ADC digitization, the collected signal is multiplied by a gain factor and is bounded to the ADC range. The electronics of the strip detectors can zero-suppress small signals while for the pixel detector only signal above the readout threshold are recorded. Tracker inefficiencies are expected due to dead or noisy pixels or dead time in electronics. Interstrip crosstalk affects resolution of clusters and tracks. In addition to the signal resulting from the charges produced in the bulk of the sensor, there is a signal induced on adjacent strips. For a charge $Q$ deposited on a single strip, some fraction $x$ (cross-talk) is registered on neighbors. These different effects were parameterized according to results obtained from detailed simulations and comparisons with CMS cosmic data taken in 2008.

\section{Geometry and Material Budget Review}

The CMS tracker geometry has been completely reviewed with the most updated information coming from the engineering's drawings. The position and orientation of the active silicon volumes and the dimensions of the passive volumes and their materials have been reviewed. Due to the large number of readout channels, the tracker detector requires a substantial amount of passive materials that are involved in the electronics, cooling or power systems. Passive materials will create unwanted effects during the detection of particles, such as multiple scattering, electron bremsstrahlung and photon conversion and an accurate knowledge of the material budget is therefore necessary. The fraction of radiation length as a function of pseudo-rapidity, seen by particles originating from the interaction point and passing straight through the tracker, is shown in Fig. 8 and Fig. 9.

The tracker weighting procedure has also been performed for each sub-detector from the smallest capacitor to the whole subdetectors. A comparison between the simulated objects and the real detector has been studied. The measured and simulated weights compatibility agrees at the 5-10 percent level.

\section{TUNING OF THE SIMULATION USING COSMICS}

In fall 2008, 6.5 million cosmic data with tracks in the tracker were taken with a magnetic field of $3.8 \mathrm{~T}$, among which 3.2 million/110000 high quality tracks passed the strip/pixel detector. These cosmic data were studied in order to tune the Monte Carlo simulation parameters. Figures 10 and 11 show the comparison between data and simulation. The deposit cluster charge depends on the impact angle of the track. A flat track will travel a longer way in the module than a perpendicular track and thus will deposit more charge on its way through the module. The cluster charge is therefore shown after incident track angle correction.

The CMS tracking resolution has been estimated after a track based alignment where the track residuals (difference between predicted and measured hit) were minimized while changing module positions. Fig. 12 shows the comparison between the simulation and cosmic data for the RMS of

\section{Tracker Material Budget}

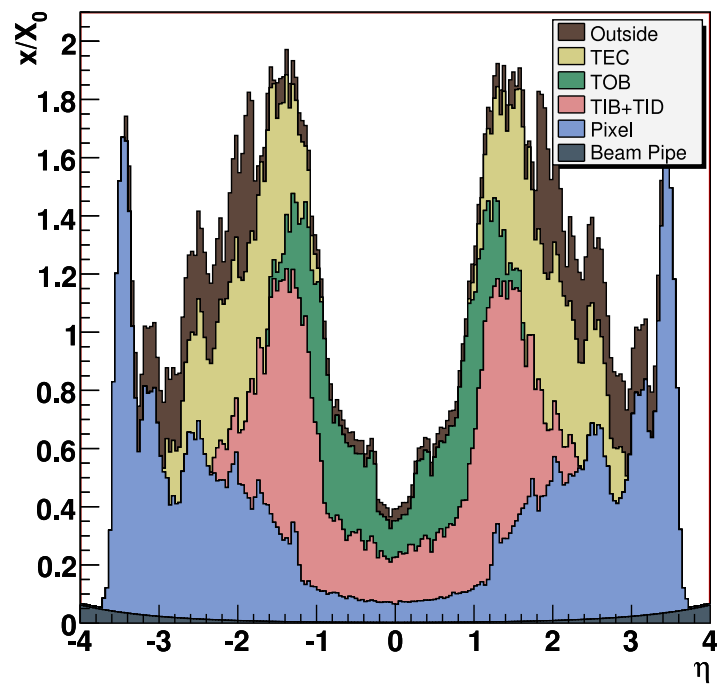

Fig. 8. Material budget profile of the tracker simulation: fraction of radiation length $\mathrm{x} / \mathrm{X}_{0}$ as a function of pseudo-rapidity $\eta$ for the different sub-detectors and structures: the beam pipe, the pixel vertex detector, the inner tracker (TIB+TID), the outer barrel (TOB) and endcaps (TEC), the outer structures (support tube, thermal screen and bulkheads).

\section{Tracker Material Budget}

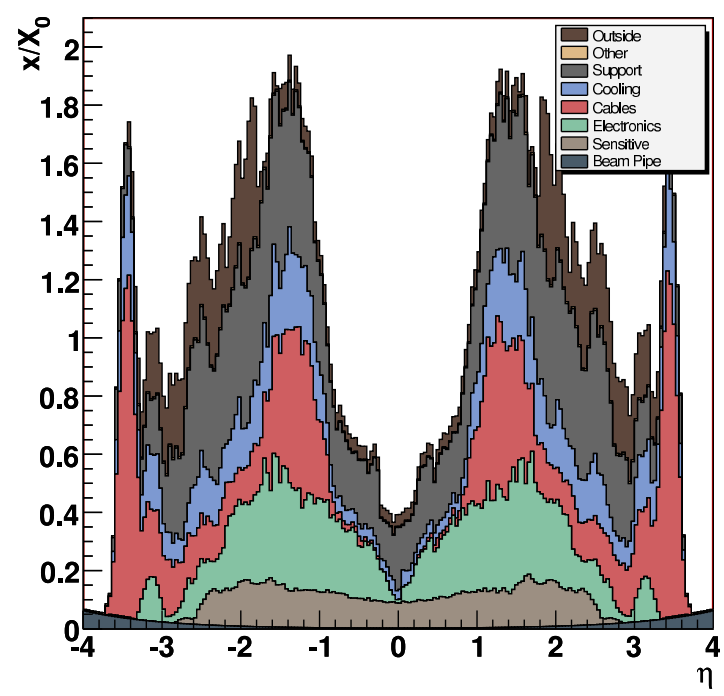

Fig. 9. Material budget profile of the tracker simulation: fraction of radiation length $\mathrm{x} / \mathrm{X}_{0}$ as a function of pseudo-rapidity $\eta$ for the different material categories: beam pipe, silicon sensitive volumes, electronics, cables, cooling pipes and fluid, support mechanics and outer structures.

residuals as function of transverse momentum for impact parameter and transverse momentum. More details about the simulation of the alignment scenarios can be found in these proceedings[8]. 

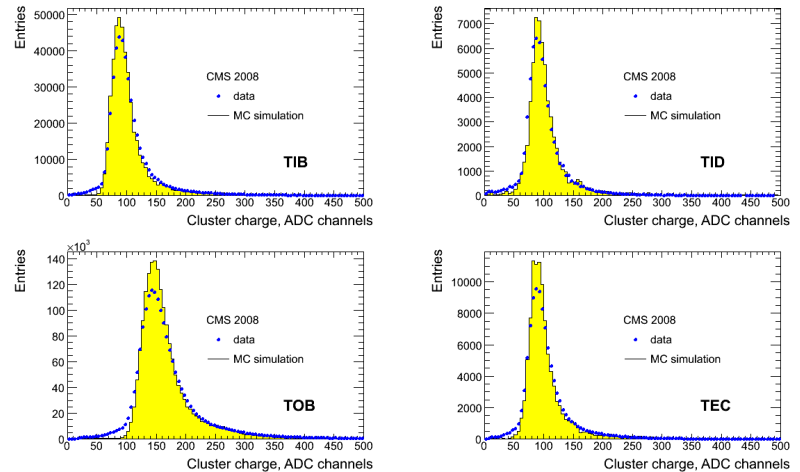

Fig. 10. Simulated and measured cluster charge for the silicon strip tracker corrected for the track incident angle. The deposited charge corresponds to the one of a track crossing the sensor perpendicularly.
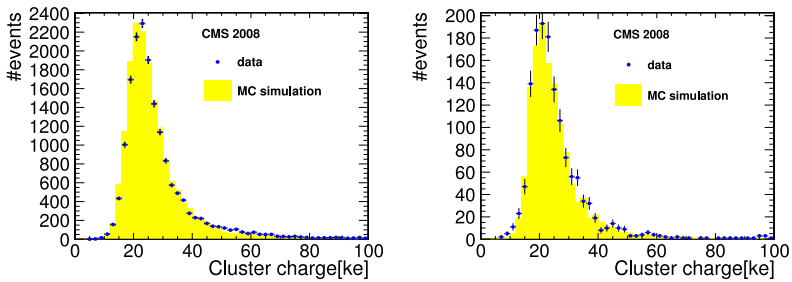

Fig. 11. Simulated and measured charge distribution for pixel barrel (left) and endcap (right).
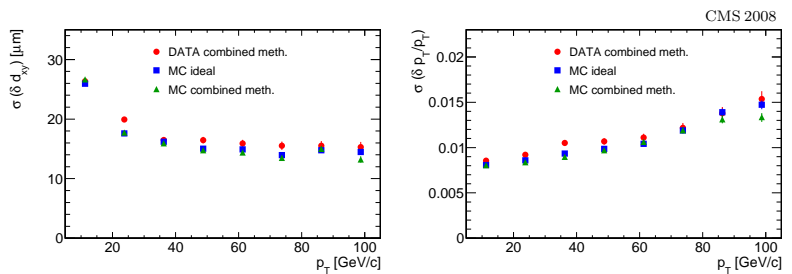

Fig. 12. RMS of residuals as function of transverse momentum for impact parameter (left) and transverse momentum (right). A combination of two methods were used to get the best alignment[8].

\section{CONCLUSIONS}

The CMS all-silicon tracker is a complex detector and requires a complex simulation. The object-oriented CMS tracker simulation based on the GEANT4 toolkit has successfully replaced its GEANT3 predecessor. It has proven robust and performant, easily extensible and configurable. The material budget has been estimated by measuring and weighing all the detector components and reproducing the correct composition of the materials of the different tracker sub-detectors. An intensive validation and tuning of the tracker simulation parameters have been done with the 6.5 million tracks that have crossed the CMS tracker in 2008 commissioning data taken.

\section{REFERENCES}

[1] CMS Collaboration, The CMS experiment at the CERN LHC, JINST 0803 (2008) S08004

[2] S. Agostinelli et al., GEANT4: A simulation toolkit, Nucl. Instrum. and Methods A506 (2003) 250-303. GEANT4 developments and applications, IEEE Transactions on Nuclear Science 53 No. 1 (2006) 270-278. Available: http://geant4.web.cern.ch/geant4.
[3] CMS Collaboration, CMS: Detector Performance and Software Technical Design Report, CERN/LHCC 2006-06, CMS TDR 8.1

CMS Collaboration, CMS Software home page. Available: $\mathrm{http}: / / \mathrm{cmsdoc} . c e r n . c h / \mathrm{cms} / \mathrm{cpt} / \mathrm{Software} / \mathrm{html} /$ General

[4] R. Brun et al., GEANT3 Users Guide., CERN Program Library W5013.

[5] M. Case and F. van Lingen, CMS NOTE-2001/057, November 2001, Detector Description Domain Architecture and Data Model

[6] G. Alverson, G. Eulisse, S. Muzaffar, I. Osborne, L.A. Tuura and L. Taylor,IGUANA Architecture, Framework and Toolkit for Interactive Graphics, CHEP03, La Jolla, California, Marh 24-28, 2003.

[7] IGUANACMS User Guide, http://iguanacms.web.cern.ch/iguanacms/userGuide.html

[8] K. Kaschube - on behalf of the CMS collaboration. Alignment of the Complete CMS Silicon Tracker IEEE 2009, Nuclear Science Symposium. 